\title{
XXIX.
}

\section{Mis,cell en.}

\section{Eine historische Notiz.}

Als ich vor Kurzem den Canstatt'schen Jahresbericht far das Jahr $1853 \mathrm{zu}$ einem anderen Zwecke durchsah, stiess ich in dem Referate aber Psychiatric auf folgende Notiz:

„Nach Boyd wurden bei Dementia paralytica nach dem Tode im Rückenmarke Resultate der Entzündung gefunden, welche auch die Ventrikel und Membranen der Gehirnbasis zu zeigen pflegten; selten wurden sie nicht in bedeutendem Grade gefunden. Gulliver fand mehrere Male bei der mikroskopischen Untersuchung Entzundungs-Körperchen im Ruckenmarkeselbst, ànlich der von Bennet in der Schrift tuber die Entzandung der Nervencentren beschriebenen. Bei der Behandlung war darauf die Aufmerksamkeit gerichtet und es wurde Liq. Hydrarg. bichl. acht mannlichen Kranken gegeben, wovon zwei sich besscrten und einer aus einem sehr hilflosen Zustande jetzt in einem Stuhle sitzen kann. Ein Anderer, sehr abgemagert, gewann in 6 Monaten 21 Pfund Gewicht mehr, zwei wurden allmälig schlechter und vier blieben stationär."

Als Quelle fur diese Notiz wird auf den Sixth general report on the district criminal and private lunatic asylums in Ireland verwiesen, den ich durch die Gate der Commissioners in Lunacy auf meinen Wunsch zugesandt erhielt, ohne dass ich indess die angezogene Stelle darin auffinden konnte. Offenbar ist die Hinweisung in dem betreffenden Jahresberichte von Canstatt. falsch und soll sie sich wahrscheinlich auf den Report of the Somerset County Asylum for 1852 bezichen. Es wäre wunschenswerth, wenn die unglischen Collegen uber die in Rede stehenden Untersuchungen Gulliver's nähere Mittheilungen machten.

Diese Notlz mag zugleich Herrn Dr. Meschede (vergl. dessen Mittheilung in No 8 der "Deutschen Clinik" dieses Jahres) zur Beruhigung dienen. Derselbe wcist nämlich seit Kurzem wiederholt und in so auffalliger Weise darauf hin, dass er zucrst eine von der grauen Degeneration verschiedene Form der Ruckenmarksaffection in einem Falle paralytischer Geisteskrankheit beobachtet habe, dass mir cine kurze Erwiederung geboten scheint, nicht etwa, um gewissc Anspruche seinerseits zu bekampfen, was von gar keinem allgemeineren Interesse ware, sondern um einer irrthumlichen Auffassung ent- 
gegen zu treten, worauf offenbar diese, mit einer gewissen Ostentation in den Vordergrund geschobenen Anৎpruche basirt sind.

Der genannte Autor hatte im Januar 1866 in Form einer ,Vorläufigen Mitthellung" den Abriss eines Falles von allgemeiner Paralyse veroffentlicht, in welchem bereits makroskopisch cine Veranderung der Hinterstrange des Rückenmarks sichtbar gewesen war; dio mikroskopische Untersuchung des fuschen Praparat's liess zahlrciche Kornchenzellen in den Hinterstràngen erkennen, auch waren die Nervenfasern zum Thell, in dem conus medullaris aber zum grossten Theil, , in thre Elemente zerfallen und in eine mehr weniger feinkornige Masse umgewandelt; ${ }^{*}$ ) ahnluches fand sich in einem Theile der Seitenstrange. Herr Mescbede sieht nun in disem Befunde vorwiegend „Veranderungen der Zwischensubstanz von mehr a cutem Character" und stellt Ihn deshalb den Fallen grauer Degeneration gegenuber. Hierauf ist Folgendes zu bemerken.

Seit lange sind unter der Bezeichnuug der grauen Degeneration auch Fälle bekannt, welche eine ahnliche, schon makroskopisch sichtbare Veranderung der Farbe und Consistenz der Hinterstrange, wie sie eben zu der genannten Bezeichnung Veranlassung gegeben, darbieten und bei frischer Untersuchung ene grosse Anzahl Fettkornchenzellen in dem veranderten Gewebe erkennen lassen; an erharteten Schnitten constatirt man zugleich cinen ausgedehnten Schwund von nervosen Elomenten. Der Reichthum an Fettkornchenzellen ist in solchen Fallen oft derart, dass schon makroskopisch in der schmutzig graulichen Substanz der IInterstrange sich gelblich weissliche, zum Theil oft baumformig-ramificirte Stellen bemerkbar machen, welche eben den - zum Thell langs der Gefassramificationen - in grosser Menge angehauften Fettkornchenzellen cntsprechen. Der von Herrn Meschede veroffentlichte Fall num liess gleichfalls bereits makroskopisch eine deutliche Veranderung der Farbe und Consistenz der IInterstrange erkennen, wahrend zugleich mikroskopisch grosse Mengen von Fettkornchenzellen gefunden wurden. Wenn die Consistenz als weich, das Ausschen des Malks als ein trubes, unregelmassig gefarbtes mit hlassgelblichem Farbenton beschrieben wird, so glaube ich nicht, dass Herr Meschede hierauf besonderes Gewicht zu legen gedenkt, da er selbst theils aus eigener Erfahrung, theils aus den in der Literatur der grauen Degeneration beschriebenen Fallen sehr gut wissen wird, wie wechselnd die Nuancen des aussern Ansehens und die Consistenz hierbei zu sein pflegen. Worin bestcht denn nun aber das Neue in scinem Falle? - Er selbst sagt es uns: es handelte sich dabei vorwegend um Veranderungen der Zwischensubstanz von mehr , acutcm" Character und die "Destruction der Nervenfasern war grosstenthels crst eingcleitet, nur an vereinzelten Stellen bis zum worklichen Verfall gediehen". Und wodurch wird dies bewesen? Man sollte meinen, dass zur Begrundung einer solchen Bchauptung wenigstens eine Untersuchung am erharteten Praparate vorgenommen ware, indess davon ist nicht die Rede, und auf die Menge der zu Grunde gegangenen Nervenfasern wird einfach durch die Untersuchung frischer, unter das Mikroskop gebrachter Stickchen des Ruckenmarks geschlossen. Sollte es Herrn Meschede

*) Wie Herr Moschede sich uberzeugt hat, dass es sich wirklich um ,in ihre Elemento zerfillene Nervenfasern" gehandelt ist nicht angegeben. 
wirklich unbekannt sein, wie weng man aus einer derartigen Untersuchung auch nur annahernd auf die Menge der geschwundenen resp. crhaltenen Nervenrohren schliessen kann? - Abrr selbst vorausgesetzt, er hatte erhärtete Sehnitte untcrsucht, war er berechtigt, einen etwa daber gefundencn geringeren Verlust an nervosen Elcmenten heranzuzichen, um einen acutereu Character, des Krankheitsprocesses daraus herzuleiten? Weiss er nicht, dass ein im Verhaltniss zu den oft eminent chronischen Krankheltssymptomen nur geringer Schwund von Nervenrohren nicht selten bcobachtet wird? - Sollte or aber auf die Körnehonzellen zum Beweise der Acuitat des Prozesses das Hauptgewicht legen, so bedarf es wohl nur der Erinnerung, dass ein solcher Schluss ein vollkommen willkuhricher ist. -- Aus dem Gesagten geht hervor, dass Herr $\mathrm{Meschede}$ keine nouen $\mathrm{Thatsach}$ en beobachtet, sondern nur einer bekan $n$ ten und noch dazu von ihm unvollstandig untersuchten Veranderung eine willkührliche $\mathrm{D}$ e u $\mathrm{t}$ u $\mathrm{g}$ gegeben hat; die cigentlichen Differenz- und Beruhrungspunkte der verschiedenen bei den paralytischen Geisteskranken vorkommenden Veranderungen des Rúckenmarks hat er nicht gekannt. Da nun aber Herr Meschede bei Hervorhebung seiner Anspruche sich besonders gegen mich wendet, der ich ilhm gegenuber gar keine solche gemacht, und grossen Werth auf diese Dinge legt, so kann es ihm nur erwunscht sein, darauf hingewiesen zu werden, dass auch unter meinen, bereits 1864, also vor seiner Beobachtung veroffentlychten Fallen von graucr Degeneration bei Paralytikern, gerade bei cinem cine cnorme Menge von Fettrornchenzellen in den IIinterstrangen gefunden wurde, $\left.{ }^{*}\right)$ wallrend beiliufig gesagt von einem acnten Verlaufe der Symptome wenigstens keno Rede war.

Ist es num abcr nicht wunderbar, dass Ilerr Meschede ir der Veborschrift za dom jungst reroffentlichten Falle (1)eutscho Climk No, 8 1868) von cincm "Falle chronischer Mychtis" spricht, trotzdem or un dieser sclben Mittheilung die besprochenen Verandernngen gerade als solche von mohr a cutem Character ganz besonders betont und darm grade das Neue sicht? Die Losung dieses Widerspu uches muss ıhm sclbst nberlassen heıben -

Wemn or ferner Gewjelt darauf legt, dass dic Ruckenmarksaffectoon in scinem 1866 publicirten Falle erst nach entwickelter Gestesstorung anfgetreten sei, so sollte or, falls or meine letzton so vielfach von lhm erwälnten Arbeiten aufmerksam gelesen, jetzt ebenso gut wissen als ich, wie wenig sich ans dem Fehlen motorischer und sensibler Storungen auf das Nichtvorhandenscin niner Erkrankung des Ruckenmarks schliessen lässt; letztere kann bereits in hohem Grade entuickelt scin, ohne dass erstere beobachtet wurden und es wird daher das erste Auftreten motorischer und sensibler Storungen durchans nicht mit dem Boginne des Krankhentsprozesses im Ruckenmark selbst als zusammenfallend angesehen werden dirfen. Auch in seinem Falle muss es also ganz dahin gestellt bleiben, ob nicht die Ruckenmarksaffection vicl fruher begonnen, als er selbst es glaubt, und bevor die motorischen Storungen anftraten

Weiter meint IIcrr Meschede, Angesichts des namentlich von mir auf diese Ruckenmarks-Affection gelegten Gewichtes darauf aufmerksam

*) S. Beob. 7, Allgem. Zeitsclir f Psych. XXI. 1864 j. 387; vgl. weitere Falle in Virchow's Archuv Bd. 38 und 39, p. 114 und 386, Beob. B und F. 
machen zu mussen, dass eine „exclusive Correlation-zwischen der beregten Ruckenmarksaffection und der paralytischen Geisteskrankheit" nicht besteht; ich erlaube mir daher gleichfalls, ihn darauf aufmerksam za machen, dass ich nun und nimmer eine solche ,exclusive Correlation" behauptet habe und auch ungemein thöricht hatte sein müssen, so etwas zu thun; ich habe sogar bestimmte Krankbeitsformen, in welchen ich ahnliche Veränderungen vermuthete, im Auge gehabt und auch andere Collegen darauf hingewiesen. Nur pflege ich mich allerdings nicht uber Dinge anszusprechen, die ich noch nicht genauer untersucht habe.

Herr Mesche de schliesst endlich mit einem Citate von Herrn Dr. Simon, durch welches er hervorheben will, dass es fraglich sei, ob die Motilitatsstörungen der Extremitäten bei Dementia paralytica wirklich auf die Körn chenzellenentwickølung zurukzuführen sind, indem sich letztere auch sehr reichlich in Fällen findet, wo keine Verainderungen des Ganges bemerkt worden, und in Fallen mit ausgesprochener Motilitatsstorung der unteren Extremitäten sich wiederholt nur mässig viele Körnchenzellen nachweisen lassen." - So hingestellt, ist hierbei gar nichts fraglich, denn ich habe ausdrucklich nachgewiesen, dass der Grad der (erkennbaren) anatomischen Veranderungen in keinem direkten Verhältnisse zur Motilitätsstorung steht; ${ }^{*}$ ) durch das genannte Citat aber muss, nach allem Vorangegangenen, in dem Leser der Glaube erweckt werden, dass ich anderer Meinung bin, wahrend ich gerade die in Rede stehende Thatsache ganz besonders betont habe.

Ich breche hicr ab und kann nur wunschen, dass Herr Meschede an meine Arbeiten nicht weitere Irrthumer kunpfe, nur nm diese nicht Platz greifen za lassen, habe ich das Wort genommen. Gewiss steht es Jedem frei, auch einen einzelnen bcobachteten Fall in Form einer „Vorläufigen Mittheilung" zu veroffentlichen und es ist das schliesslich Geschmacksache; knupfen sich aber bei gleichzeitiger Verschweigung anderer Thatsachen allgemeine Folgerungen daran, so erwachst das Recht und die Pflicht dem entgegenzutreten. W.

Berlin, im April 1868.

*) So heisst es z. B. in meinem Aufsatze (Archiv fur Psychiatrie und Nervenkrankheit I. 1. p. 88): „es gibt, wie ich gezeigt habe, Fille von sehr entschieden myelitischer Erkrankung der Seiten- und Hinterstrange, ohne dass im Leben die Motilitat wahrnehmbar beeinträchtigt ist" $u$. s. w.

Notiz. Da dieses Heft schon sehr umfangreich geworden ist, mussen wir die Fortsetzung der Protocolle der Berliner medicinisch-psychiologischen $\mathrm{Gc}-$ sellschaft auf das dritte Heft verschieben.

Druckfehler. Seite 378 Z. 11 v. u. liess "der" anstatt "des", Seite 383 Z. 6 v. u. liess ,,vom Krampfe" anstatt ,von Krimpfen". 